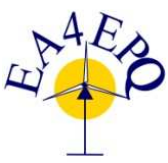

International Conference on Renewable Energies and Power Quality (ICREPQ'13) Bilbao (Spain), 20 ${ }^{\text {th }}$ to $22^{\text {th }}$ March, 2013

Renewable Energy and Pourer Qualiny. Fournal (RE\&PQJ)

ISSN 2172-038 X, No.11, March 2013

\title{
Performance characterization of a PV system using wavelet transform and genetic algorithm
}

\author{
P. Janik ${ }^{1}$, P. Kostyła, ${ }^{1}$, J. Rezmer ${ }^{1}$,J. Szymanda ${ }^{1}$, T. Sikorski ${ }^{1}$, Z. Wacławek ${ }^{1}$, D. Lehmann ${ }^{2}$ \\ ${ }^{1}$ Department of Electrical Engineering \\ Wroclaw University of Technology \\ Wyb. Wyspianskiego 27, 50-370 Wroclaw (Poland) \\ Phone number:+0048 3202901, e-mail: przemyslaw.janik@pwr.wroc.pl \\ ${ }^{2}$ Faculty of Mechanical, Electrical and Industrial Engineering \\ Brandenburg University of Technology, Cottbus \\ Walther-Pauer-Str. 5, 03046 Cottbus (Germany) \\ Phone number: +0049355694032
}

\begin{abstract}
This paper presents a study of PV system performance in terms of power quality. Wavelet transform has been applied as a tool for signal characterization and future extraction. A Gaussian curve has been proposed as an approximation of daily production. The parameters of the curve were computed using genetic algorithm.
\end{abstract}

\section{Key words}

photovoltaic system, wavelet transform, energy production curves, genetic algorithms

\section{Introduction}

The anticipated shortage of fossil fuels and concerns about environmental pollution strongly reinforce the use of renewable energy sources. Green energy production technologies are now competitive with traditional solutions based on fossils.

Numerous forecasts indicate an increasing and overwhelming contribution of solar energy to the global energy mix in the future. Annual reports of EREC or Greenpeace and German Advisory Council on Global Change [1] are justifying examples.

The proliferation of PV systems into the power grid causes new challenges for the transmission and distribution of energy [2], which are unique for distributed generation. Power quality, system security, monitoring and control strategies are subjects of on-going research activities.

One of the research issues is the power output variation in PV systems. Assumed is a daily production proportional to the theoretical irradiation. Due to overclouding, shadows and internal failures the energy production curve is often significantly different. There is no match between the real and theoretical values of energy production. This paper is an attempt to characterise and quantify this discrepancy. Firstly, the photovoltaic system used for the collection of real production data has been described.
The wavelet transform was considered an appropriate tool for the smoothening of production curves and for peaks extraction as well. A conscience description of discrete wavelet transform follows in the subsequent section.

An arbitral curve has been selected to depict the daily production pattern. Generally, a method for obtaining the daily production or consumption pattern was needed.

The coefficients have been determined by the application of optimization procedure using genetic algorithm.

New PQ indices based on the momentum of production curve have been introduced.

The authors assume that the presented approach may be also suitable for the termination of load characteristics and other production units.

\section{PV System}

\section{A. Hardware description}

The $15 \mathrm{~kW}$ Photovoltaic research system (Fig. 1) is directly connected to the power grid. Therefore it is suitable for research on the interactions between PV and the grid. Three types of panels are connected in parallel: monocrystalline, polycrystalline, and thin film (amorphous).

The system was installed on the rooftop without any tracking mechanism (Fig. 2), and with accordance to standards [4].

Every unit of each type has the power of $5 \mathrm{~kW}$ and has a separate converter feeding into low voltage grid. The efficiency depends on the produced power and in a wide power range is within the $96-98 \%$ limit. Every module is equipped with a weather station measuring the solar irradiation, temperature etc. The comparison between irradiation curve and produced power is crucial for the operational control of the system. 


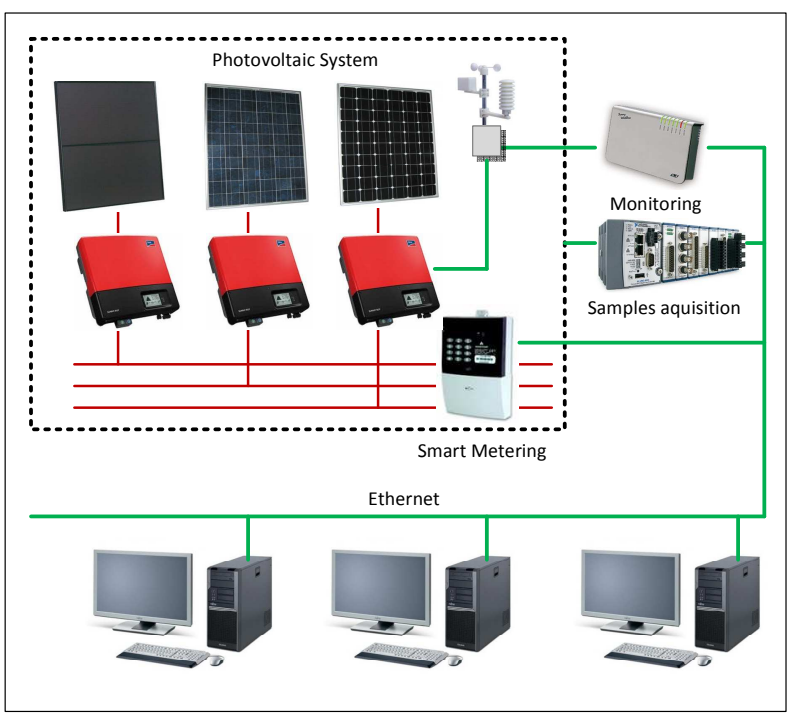

Fig. 1. Photovoltaic system used for data collection

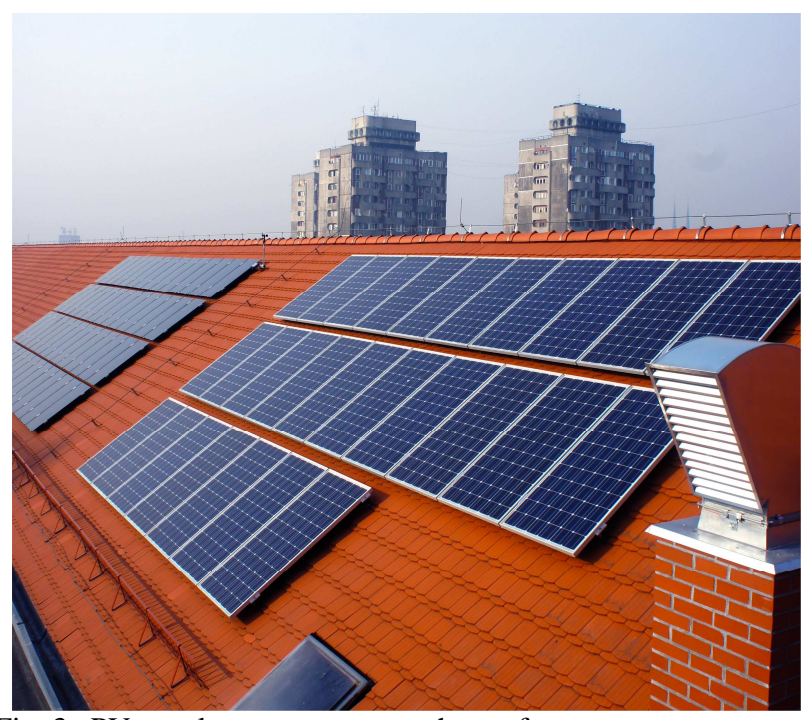

Fig. 3. PV panels arrangement on the roof

The inverters are equipped with grid management functions. Most important are displacement power factor regulation and frequency dependent active power limitation.

During normal operation the displacement power factor was set to one. No reactive power was supposed to be feed into the grid as every adjustment reduces the efficiency.

Frequency dependent active power limitation was adjusted to the requirements in [3]. During the research time no violation of the frequency limit was observed.

The inverter can slowly increase the active power output after a grid connection. The factory pre-set value $20 \%$ Pmax/sec was used. After any disturbance (DC or AC side) the active power is increased by $20 \%$ of the nominal value per second. The increase ratio can by freely changed from 1 to $100 \%$. Additionally a one minute operation with $10 \%$ of nominal power can be programmed. Neither of this possibilities was used during the recording of power curves.

After a grid incident the reconnection times and limits can be freely chosen. The values summarised in Table I have been used. The values correspond with recommendations in [3].
Constantly the I-V tracking for MPP was enabled to maximize the power output.

Table I. -Reconnection times and values

\begin{tabular}{|l|c|c|}
\hline \multicolumn{1}{|c|}{ event/limit } & time/value range & set value \\
\hline $\begin{array}{l}\text { connection time after a } \\
\text { brief system incident }\end{array}$ & $0 \ldots 1,600 \mathrm{~s}$ & $5 \mathrm{~s}$ \\
\hline $\begin{array}{l}\text { connection time after a } \\
\text { longer system incident } \\
>=3 \mathrm{~s}\end{array}$ & $0 \ldots 1,600 \mathrm{~s}$ & $60 \mathrm{~s}$ \\
\hline $\begin{array}{l}\text { connection time after a } \\
\text { restart }\end{array}$ & $0 \ldots 1,600 \mathrm{~s}$ & $60 \mathrm{~s}$ \\
\hline $\begin{array}{l}\text { minimum voltage for } \\
\text { reconnection }\end{array}$ & $70-240 \mathrm{~V}$ & $199 \mathrm{~V}$ \\
\hline $\begin{array}{l}\text { maximum voltage for } \\
\text { reconnection }\end{array}$ & $100-280 \mathrm{~V}$ & $249.5 \mathrm{~V}$ \\
\hline $\begin{array}{l}\text { minimum frequency for } \\
\text { reconnection }\end{array}$ & $44-60 \mathrm{~Hz}$ & $47.5 \mathrm{~Hz}$ \\
\hline $\begin{array}{l}\text { maximum frequency for } \\
\text { reconnection }\end{array}$ & $50-65$ & $50.05 \mathrm{~Hz}$ \\
\hline
\end{tabular}

\section{B. Monitoring System}

The research installation is equipped with two redundant monitoring systems. One is based on the LabView software and CompactRio controller (Fig. 4). The second was delivered by converter manufacturer. Data from manufacturers default monitoring have been used to enable easy results generalization.

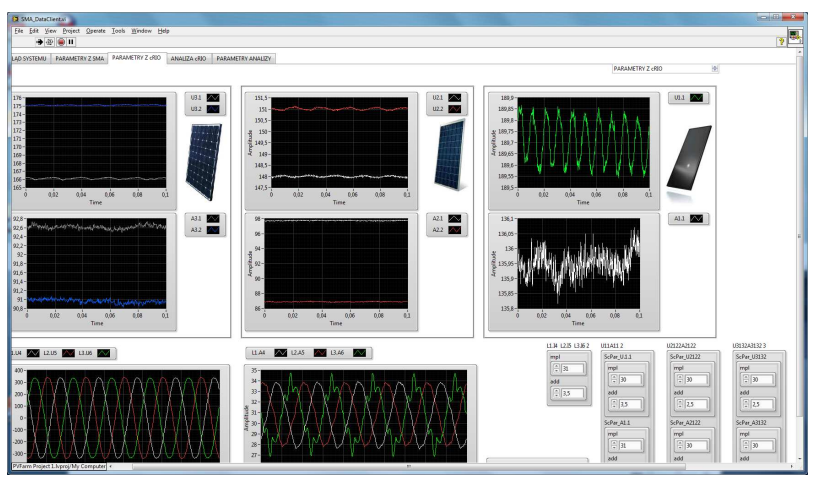

Fig. 4. PV monitoring system - view panel

The weather station monitored panels and ambient temperature, wind speed and solar irradiation.

The voltages and currents on DC and AC site have been recorded and the data was used for power and energy production computation.

All the data are available from a dedicated manufactures page with a $15 \mathrm{~min}$ raster.

\section{Wavelet transform}

There are two general perceptions of wavelet transform. Signal processing perspective allows wavelet algorithms to be considered as a filter bank composed of band pass filters [5]. The wavelets can also be introduced using a procedure called 'lifting' which is defined in the discrete time domain without the reference to filters [6].

The key feature of wavelets is the varying window, wide for low frequencies and narrow for high frequencies. This future results in an optimal time-frequency representation 
of signals. Moreover, the time detection of fast transients and signal de-noising can be performed as well.

The Continuous Wavelet Transform (CWT) of a signal is given as

$$
X_{a, b}=\frac{1}{\sqrt{|a|}} \int_{-\infty}^{+\infty} x(t) \psi\left(\frac{t-b}{a}\right) d t
$$

where $\psi(t)$ is the mother wavelet and the other wavelets are constructed as its dilated and translated versions. The translation and dilation operations are represented through parameter $b$ and $a$ respectively.

Numerical computation is usually performed using the Discrete Wavelet Transform for a selected subset of scales and positions. This scheme is conducted by using filters and computing so called approximations and details. The approximations (A) are the high-scale, low frequency components of the signal. The details (D) are the lowscale, high-frequency components. The DWT coefficients are computed using the equation:

$$
X_{a, b}=X_{j, k}=\sum_{n \in Z} x[n] g_{j, k}[n]
$$

where $a=2^{j} \quad b=k 2^{j} \quad j \in N \quad k \in Z$.

Filter $\mathrm{g}$ is the equivalent of the mother wavelet. The decomposition (filtering) process can be iterated, so that one signal (s) is broken down into many lower resolution components constituting the wavelet decomposition tree (Fig. 5).

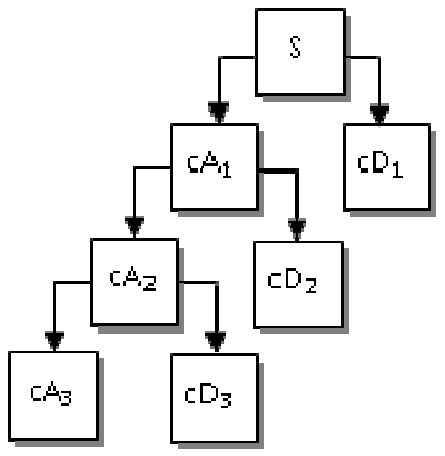

Fig. 5. Decomposition tree of DWT

Every one of wavelet transform subbands is reconstructed separately from each other, so as to get $k+1$ separated components of a signal $\mathrm{s}[\mathrm{n}]$. The MATLAB multires function [7] calculates the approximation to the $2 \mathrm{k}$ scale and the detail signals from the $2^{1}$ to the $2^{\mathrm{k}}$ scale for a given input signal. It uses the analysis filters $H$ (lowpass) and $G$ (highpass) and the synthesis filters RH and RG.

In the update scheme the equivalent operations on the signal $s[n]$ are called prediction and update (Fig. 6). The procedure can be iteratively repeated on the $s_{j-1}$ signal used as the input for the next step. The input for next steps is always reduced by half.

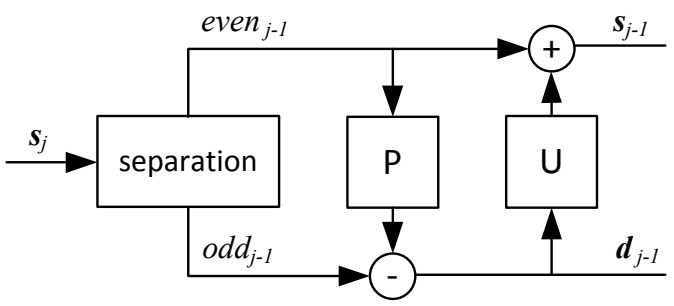

Fig. 6 Lifting operation with the Prediction and Update steps
In the prediction step some correlation between successive samples is assumed, e.g. the signal yields some structure. Knowing the value of the input signal at sample $2 \mathrm{n}$ the value at sample $2 \mathrm{n}+1$ is assumed to be identical. The difference is storied.

In the update step the value is changed with regard to the information gathered during prediction. In the simplest version it can be replaced with an average smoothening the signal.

For the smoothening operation on daily production curves the function has been used.

\section{Genetic algorithms}

For the computation of parameters of the load curves a robust optimization method was needed, therefore genetic algorithms have been selected.

Genetic algorithms represent a group of optimization methods, which imitate natural processes. They are numerical procedures based on random choices in an effective search of the optimal solution. The vocabulary and definitions are derived from biology [8].

The population members, the chromosomes, are potential solutions to a problem. The chromosomes are represented as binary sequences. Individual elements of the chromosomes are called genes.

The set of feasible points must be swept through to find the optimal solution. There is a tradeoff between application of the knowledge form already reached solutions and the fine and detailed check of the input space. Genetic algorithms offer a compromise between those two goals.

They provide a multidimensional exploration of the output values due to the changes in the potential solution population.

In every subsequent population only the best fitted members are reproduced while the remaining members are eliminated from further processing. As a criterion of the appropriateness of the reached solution an objective function is used. In subsequent iterations some chromosome satisfy the objective function better than others. A quadratic error between the measured and approximated function has been used as an objective function.

Practical implementation of the genetic algorithm starts with the setting of the first population being at the same time the first approximation of the solution. The gens were selected randomly and therefore there is little chance of reaching the best solution in the first step.

The objective function is used to value every member of the population. New members are reproduced in successive generations utilizing the rule of roulette wheel. Each member of the population is represented through a piece of the wheel, which size is determined through the objective function. A big size makes the probability of a selection for further processes much higher [9].

An offspring (a replica) is created for randomly selected member of the population which undergoes some genetic operations, being the next step in the procedure. 
The modifications are due to crossovers and mutations. Crossover results in a mutation of parental trials in chromosomes of two descendants. Genotype fragments are exchanged between two members (Fig. 7). The pair and the crossover point are selected randomly.

In a mutation operation gens can change their values (Fig. 8 ). A random value change for every gene is predefined.

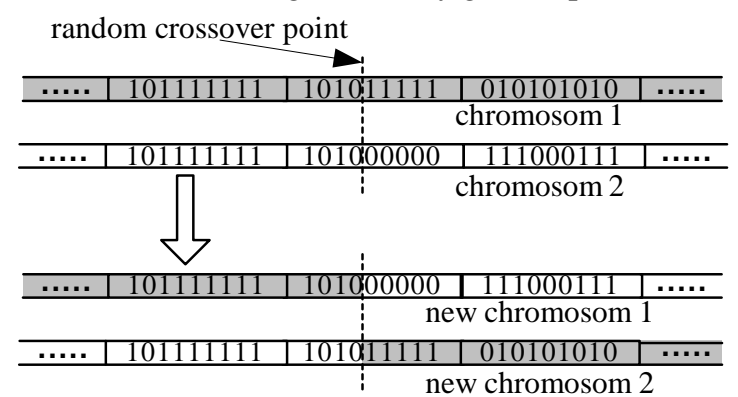

Fig. 7. Schematic representation of the cross-over operation

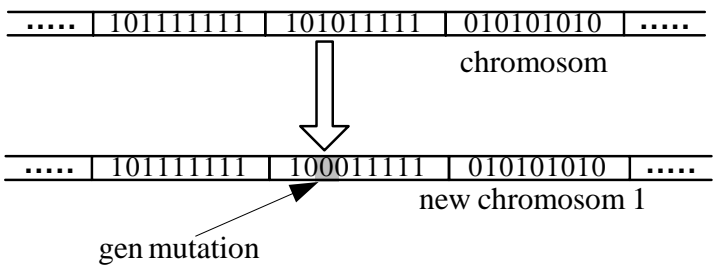

Fig. 8. Schematic representation of mutation

Optimization Toolbox GAOT [10] for Matlab was used for the calculations. The library contains subroutines for defining and selecting the method parameters, such as the evaluation function, determination of the boundary conditions for the estimated parameters, specification of the population size, settings for the closing condition, and criteria for the selection, mutation and crossover operations.

\section{Measured production curves}

Fig. 9 shows the daily power recorded over three subsequent days. Despite of a relatively long averaging period, 15 minutes, the curves reveal a certain dynamic.

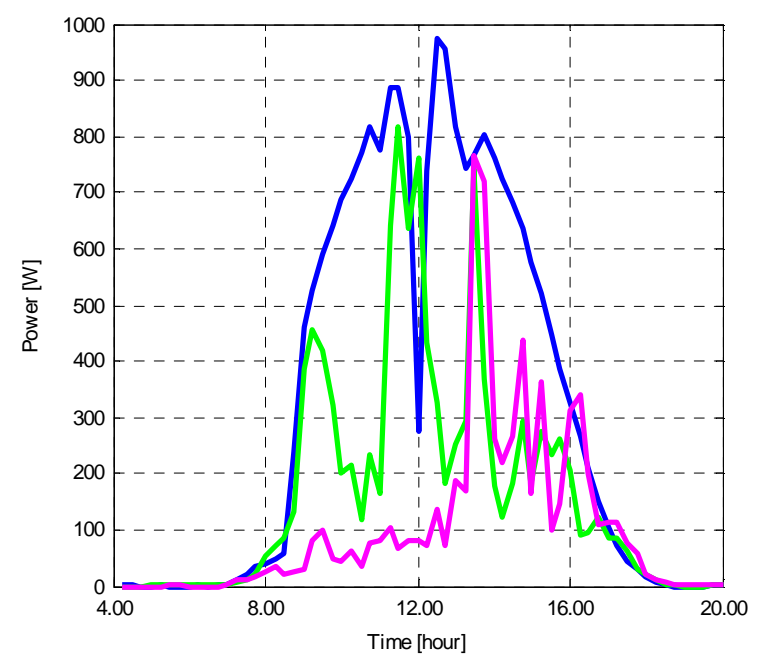

Fig. 9. PV module power on three subsequent days.
The changes correspond with the measured solar irradiation (Fig. 10) but do not correlate with the theoretical irradiation, which has almost constant shape over three days (Fig. 11).

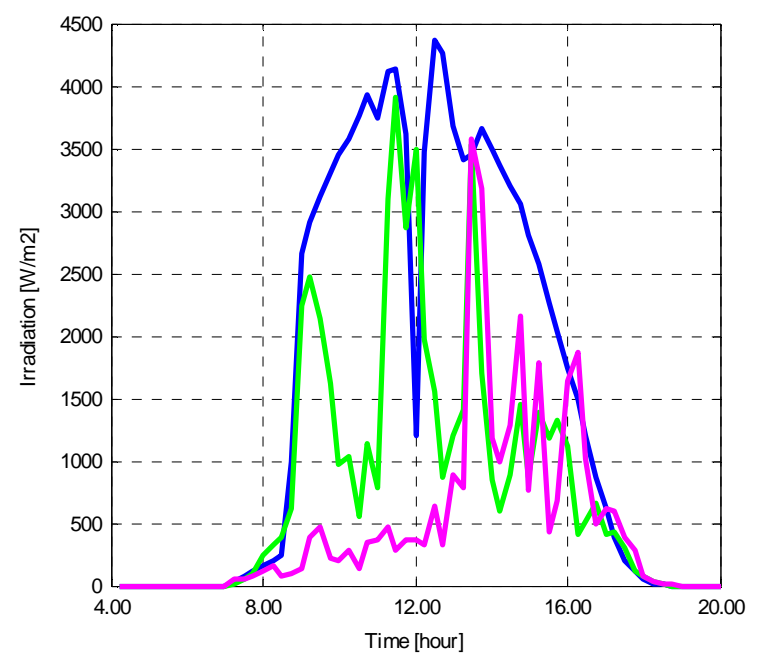

Fig. 10. Measured solar irradiation on three subsequent days

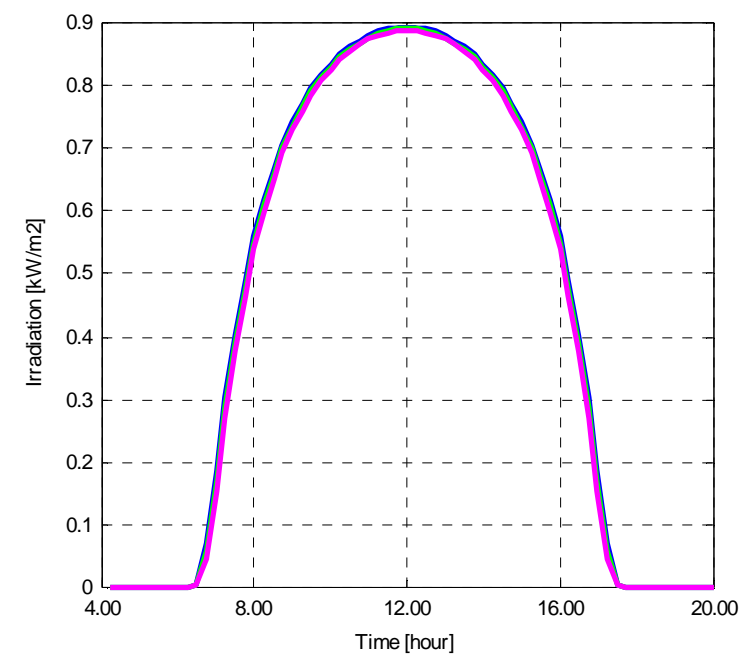

Fig. 11. Theoretical Irradiation for the location

\section{Computational results}

It is desired, that the power production in a PV system is coherent with a predefined function, e.g.:

- a constant line (possible with storage)

- covering demand curve (possible with storage)

- Gaussian curve

The aberration from that curve can be seen as parameter for the production pattern description and tariff regulation. For the production approximation an Gaussian function was used in this paper. The general expression is

$$
y(t)=\alpha_{1} e^{-\left(\alpha_{2}\left(t-\alpha_{3}\right)\right)^{2}}
$$

the $\alpha$ coefficients were computed using genetic algorithm. The coefficients are summarised in Table 1. It is visible that the daily production curve can be given with only three numbers. As an objective function for the optimization procedure the least squared error between the approximated and measured curve has been selected. 
Table 1. Coeffcients of curve approximations

\begin{tabular}{|c|c|c|c|}
\hline Curve no. & $a_{1}$ & $a_{2}$ & $a_{3}$ \\
\hline 1 & 974,16 & 0,082 & 33,42 \\
\hline 2 & 539,72 & 0,088 & 32,01 \\
\hline 3 & 362,3 & 0,099 & 41,34 \\
\hline
\end{tabular}

The curves with the parameters in Table 1 are given in Fig. 12, Fig. 13 and Fig. 14.

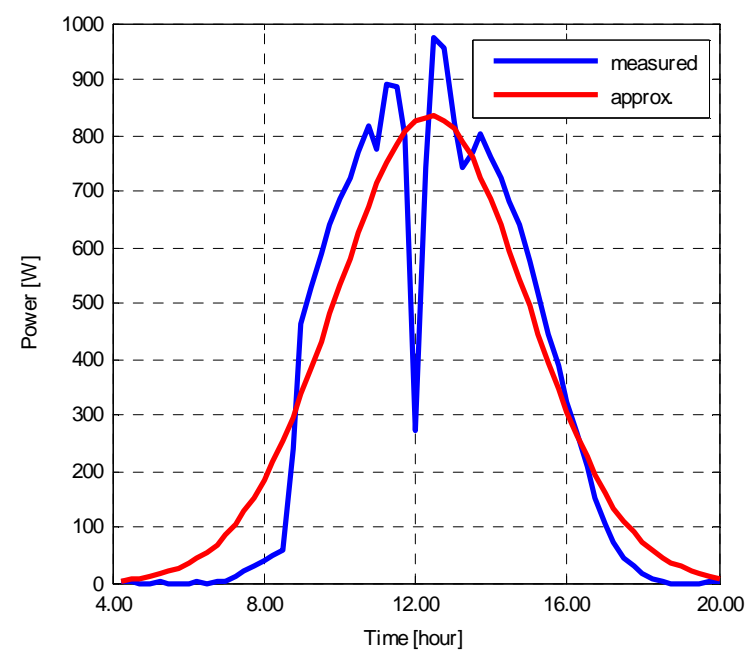

Fig. 12. Gaussian approximation of daly power curve

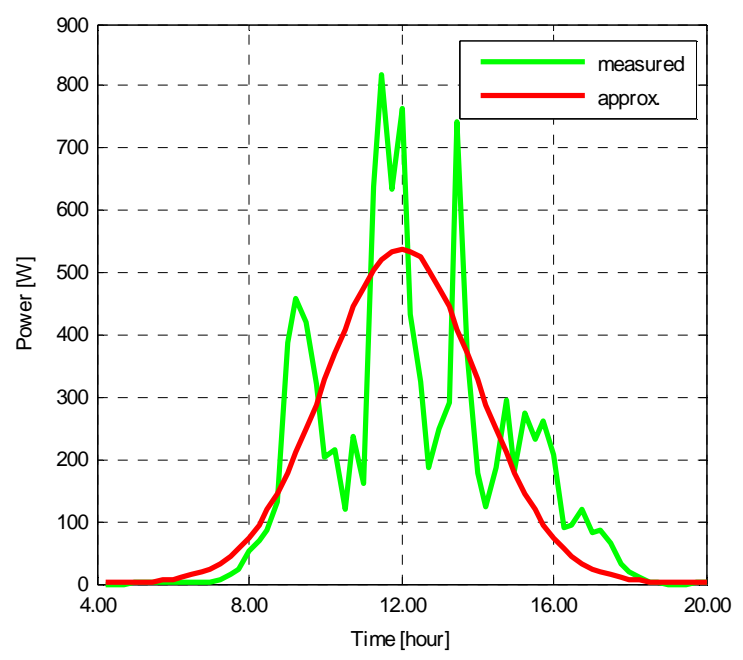

Fig. 13. Gaussian approximation of daly power curve

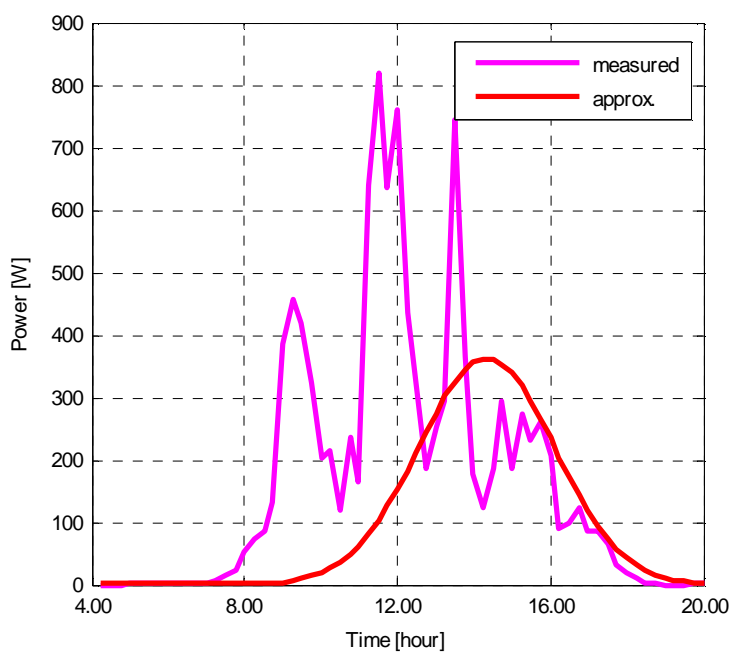

Fig. 14. Gaussian approximation of daly power curve
The example in Fig. 14 was selected deliberately, as a case where the distortion of the power curve is so significant, that an optimal approximation is not satisfactory. Sparation of dips with the wavelet transform was proposed. Three different wavelts (wspline, daub and maxflat) wer used. The resutls (Fig. 15) give almost the same approximated curve as in Fig 12.

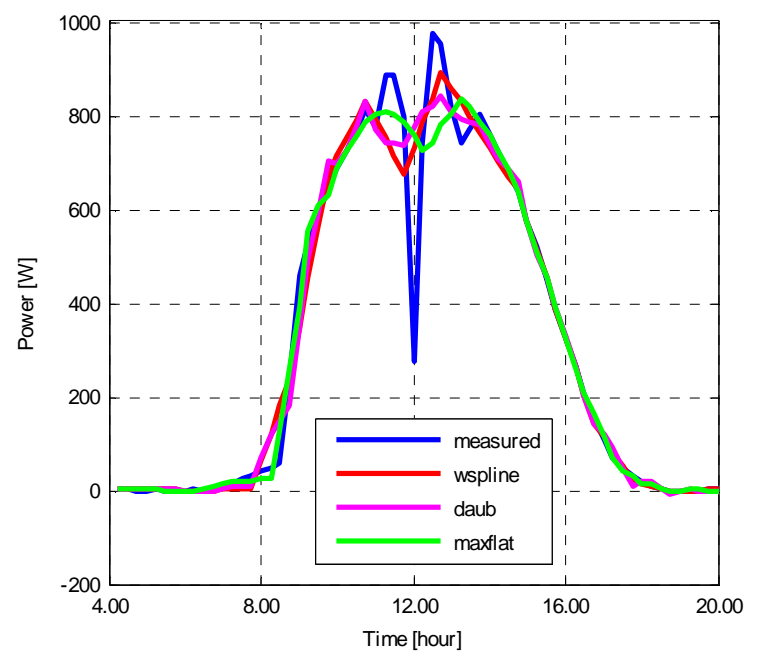

Fig. 15. Wavelet transform applied for smoothening

The details, e.g. separated rapid changes in the power curve, can be seen as an additional power quality index for the power production. Precisely, the dips amplitude and its number over a day are proposed as an index.

Fig. 16 presents the separation of the dip in Fig. 12 and Fig. 15 with three different wavelet functions.

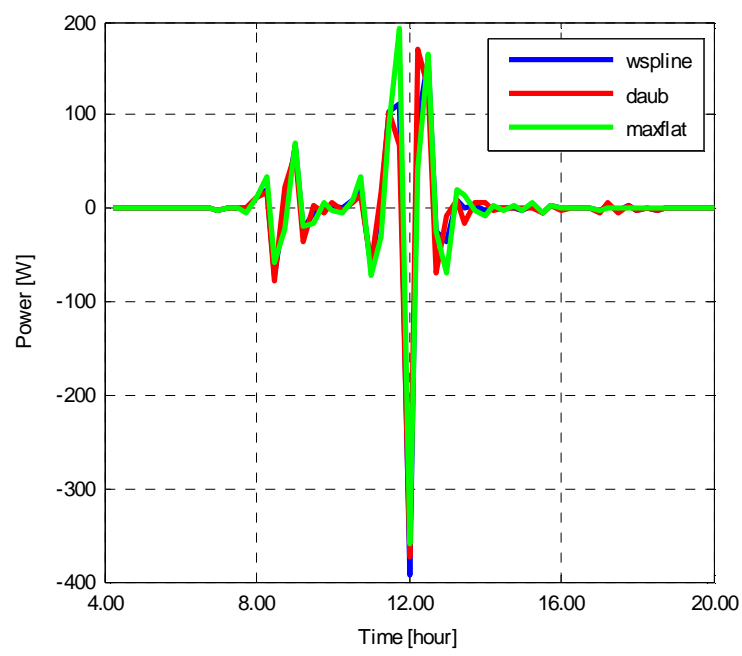

Fig. 16. Peaks separated from power curve

The amplitude and position of the dip was coherently indicated by every method.

\section{Conclusion}

The number of PV installations is growing rapidly. The daily energy production differs significantly from the theoretical irradiation curve due to wheatear conditions and disturbances. Especially unwanted are rapid and unpredictable changes in the power production. 
For the assessment of daily power curves used for PV system characterization and tariff construction a description utilizing Gauss curve was proposed. Only three parameters are sufficient to characterize the curve.

Moreover, the wavelet transform was used to separate the approximation and details. That means, to separate the smoothed PV power curve from rapid changes in the production.

The separated rapid changes can be interpreted as a power quality index summarizing the information about dip amplitude and frequency of occurrence in a given time period.

Further research is needed on power curves measured with higher sampling range in the interval between 10 minutes and 200 milliseconds. It would help to understand the nature of changes in the production of power in PV systems.

\section{Acknowledgement}

The authors would like to thank the Polish National Science Centre for financial support under Grant DEC2011/01/B/ST8/02515

\section{References}

[1] World in Transition. A Social Contract for Sustainability" German Advisory Council on Global Change (WBGU), Berlin 2011

[2] .H. Tanaka and K. Yamashita, "Photovoltaics: Developments, Applications and Impact", Nova Science Publishers 2010, pp. 249-281

[3] VDE-AR-N 4105 Generators connected to the low-voltage distribution network-Technical requirements for the connection to and parallel operation with low-voltage distribution networks [4] A. Bergman, "Photovoltaicanlagen", VDE Verlag 2011

[5] M. Vetterli, C. Herley. "Wavelets and Filter Banks: Theory and Design", IEEE Trans. on Signal Processing. 1992; 40: 2207-2232

[6] W. Sweldens, "The lifting scheme: A custom design construction of biorthogonal wavelets", Applied and Computational Harmonic Analysis, Vol. 3, 1996, No. 2, pp.186-200

[7] A. Jensen, A.laCour-Harbo, "Ripples in Mathematics. The Discrete Wavelet Transform", Springer-Verlag, Berlin, 2001

[8] David E. Goldberg, "Genetic Algorithms in Search, Optimization and Machine Learning" Addison-Wesley Pub. Co. 1989

[9] M. Wael, AL-Hasawi, Khaled M.EL-Naggar, "A Genetic Based Algorithm for Digitally Recorded Impulse Parameter Estimation"; IEEE PowerTech. conf. IEEE Bologna, Italy, June 2003

[10] Ch. Houck, J. Joines, M. Kay, "A Genetic Algorithm for Function Optimization: A Matlab Implementation" Technical Report: NCSU-IE-TR-95-09, North Carolina State University, Raleigh, NC, 1995 